\title{
Las vías fluviales, infraestructuras y puertos: la industria del contenedor, sus aportes al transporte multimodal, visión en Colombia $\stackrel{\Perp}{\Perp}$
}

\author{
Waterways, infrastructure, ports: container industry and their contribution to multimodal transport: \\ a Colombian point of view
}

Leslio Ricardo González ${ }^{1 *}$, Adm. Negocios Internacionales.

${ }^{1}$ Corporación Universitaria Remington; Diplomado en transporte marítimo, fluvial y legislación portuaria, Sociedad de Ingenieros del Atlántico y Pronaval; Diplomado Visión Competitiva, Desafío Empresarial y Solidario, Universidad Fitec y Funcolsub; Participante en la construcción del plan decenal de educación 2006/2016; uso de buen retiro de la Armada Nacional de Colombia. Vicepresidente de Cordesab.

(Recibido: 16 de octubre de 2013; aceptado: 19 de noviembre de 2013).

\section{Resumen}

Los ríos conforman un sistema de circulación lineal, vectorial, jerarquizado y estructurado para trasladar sedimentos y fluidos vitales a través de las Cuencas Hidrográficas y sus desembocaduras, realizando complejas reacciones dinámicas, mecánicas, energéticas, químicas y bioquímicas con el propósito de dar sustento en todo su recorrido a la vida en sus diferentes formas. Constituyen una fuente de riqueza para la conservación de especies animales y vegetales. Tienen una biodiversidad que es importante para los ecosistemas de las regiones litorales, proporcionando alimento por medio de la pesca, agua potable, mueven las turbinas para producir energía eléctrica, riego a las regiones secas, se utilizan en navegación y sirven para recreación. La navegación permitió que los pueblos que habitaban las riberas de los mares y grandes ríos pudiesen conectar unos con otros, rompiendo de esta forma el aislamiento progresivo que había supuesto la expansión de los seres humanos en el planeta. Además del comercio, algunos pueblos vieron la utilidad de usar las naves para ejercer un poder militar e influir políticamente en otros pueblos. El transporte fluvial tiene sobre el ferroviario y el carretero ventajas fundamentales; requiere bajo costo de inversión y mantenimiento, bajo consumo de energía, gran capacidad de manejo de carga en los puertos y gran capacidad de tracción para recorrer grandes distancias.

Palabras clave: competitividad, optimización, transporte.

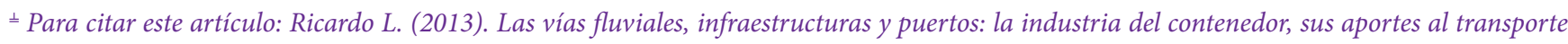
multimodal, visión en Colombia. Rev Humanismo y Sociedad. Volumen 1: 162-167

* Autor para correspondencia: Leslio Ricardo González. Corporación Universitaria Remington. E-mail: lesrigon@yahoo.com.
}

Revista. Humanismo.Soc. 2013; Volumen 1: 162-167. 


\section{Abstract}

Rivers form a system of linear and vector movements, which are hierarchical and structured to move sediments and vital fluids through watersheds. They make complex dynamic, mechanical, energetic, chemical and biochemical reactions to support life in its various forms throughout their route. Rivers are a vital resource for the conservation of plant and animal species. They foster biodiversity in coastal ecosystems and provide food through fishing. They also provide drinking water and move the turbines to produce electricity. They supply irrigation to dry areas as well as navigation and recreation. Navigation allowed peoples inhabiting the shores of the seas and large rivers connect with each other, thus breaking the isolation caused by the progressive expansion of humans on the planet. Besides trade, some peoples used ships to increase military power, and gain political influence on others. River transport has fundamental advantages over railroads and highways: it requires lower investment and maintenance cost, lower power consumption, and they have larger cargo capacity at ports and plenty of traction to go the distance.

Key words: competitiveness, optimization, transport.

\section{Introducción}

El transporte fluvial, que estuvo a punto de desaparecer como medio de transporte de importancia a mediados del siglo XX, se revitalizó gracias a la posibilidad de transporte intermodal que ofrecen los contenedores, que facilitan la carga, descarga y almacenamiento de grandes cantidades de mercancía heterogéneas. El transporte de viajeros tiene un carácter regular en trayectos entre dos puertos del mismo río, debido a la creciente importancia de los servicios de transporte en el comercio nacional e internacional y como elemento fundamental del desarrollo de los países, la tendencia internacional es hacia el multimodalismo de todas las operaciones involucradas en la cadena logística de un solo contrato de transporte a fin de optimizar el servicio y aumentar la competitividad de los bienes tranzados, esto implica lograr que los sistemas de transporte terrestre, férreo, fluvial, marítimo y aéreo funcionen de manera óptima y articulada. (Antonioz Transporte Intermodal) (Figura 1).

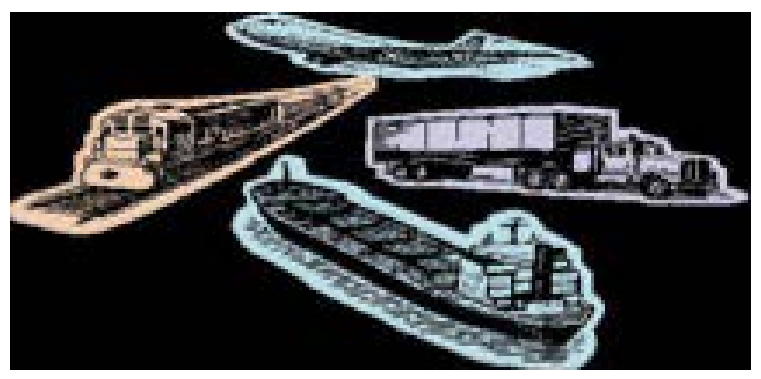

Figura 1. Modos de transporte, articulados y administrados eficaz y eficientemente, generan el sistema multimodal. Fuente: Lógico 4576 logística/ lenguaje de transporte intermodal
Es bien sabido que para cargas pesadas y para contenedores las vías fluviales son la mejor alternativa, tanto en razón de costos como por un menor impacto ambiental. Los barcos de contenedores provocaron un cambio tecnológico en el manejo de la carga y vincularon la industria del transporte ferroviario y por carretera con la navegación oceánica y Fluvial, para ello se debe contar con ríos navegables y con una infraestructura portuaria adecuada. (Figura 2) (Antonioz Puertos y Transporte Intermodal). A pesar de la importancia de este modo como medio de transporte de carga y como base de desarrollo del comercio nacional y/o internacional, Colombia que posee unas grandes ventajas comparativas a nivel global, algo paradójico su estado de atraso o subdesarrollo económico, entre estas ventajas tiene las cuencas hidrográficas.

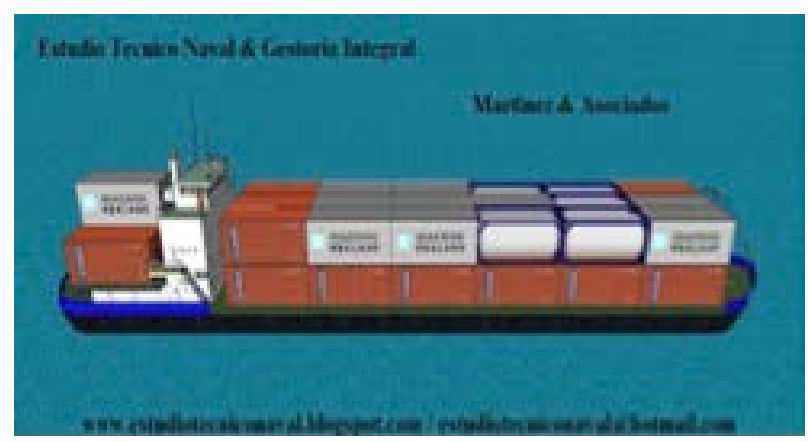

Figura 2. Buque portacontenedores fluvial o aguas interiores. Fuente: Diseño M\&B Elite Naval Design Study Industria Argentina 
El trasporte intermodal no se podía usar eficientemente en ningún lugar del mundo, hasta los años 70/80, había problemas operativos, muchos marcos regulatorias, faltaban políticas. El país que más invierte en la importancia del transporte en la economía, EE.UU crea el contenedor, originando la primera solución a la problemática de este modelo de transporte reducción significativa en los costos, y uso masivo del contenedor, fue la base de la logística moderna y posibilito la globalización. Logística es una gestión global, para obtener el mayor rendimiento en las cadenas de abastecimiento, producción, comercialización y distribución, para asegurar el abastecimiento de insumos o productos básicos en el momento preciso, con el embalaje/unitarizacion adecuada, para permitir el manipuleo adecuado, el transporte más adecuado según valor/tiempo, haciendo solo el almacenaje imprescindible, buena gestión de inventario (stock)/ donde/ cuanto, todo esto para reducir al máximo los costos y obtener el mejor precio posible. (Orellac Rogelio I Foro Global Contenedores).

El objetivo principal del sistema intermodal es combinar de la manera más eficiente el uso de los distintos modos disponibles, para eso se requiere de adecuadas infraestructuras, carreteras, ferrovías, hidrobias, puertos, terminales, tecnología, legislación, y lo más importante talento humano capacitado. (Antonioz Transporte Intermodal)

\section{Visión en Colombia}

El Sistema Fluvial de Colombia se encuentra repartido en cuatro cuencas mayores y una región de cuencas menores, los principales ríos de este Sistema Fluvial representan una longitud total de $24.725 \mathrm{Km}$. de los cuales son navegables permanentemente $7.063 \mathrm{~km}$ y con los tramos navegables en parte del año, suman $18.225 \mathrm{Km}$. a pesar de la magnitud geográfica de nuestro Sistema Fluvial ,el Sistema de Transporte de Colombia está gravemente sesgado a favor del Transporte terrestre, por carreteras (Ospina Hernández Mariano).

En Colombia el proceso de navegación fluvial se hace en gran parte de forma empírica, gracias a la experiencia y habilidad de veteranos capitanes al servicio de las empresas navieras, quienes por años y generaciones han recorrido nuestros ríos memorizando sus canales. El movimiento de contenedores a nivel mundial viene creciendo fuertemente, año tras año, desplazando otras vías más tradicionales de carga y transporte. En el país, el transporte de carga de contenedores se inicia en el 2004, cuando la empresa Sofasa, haciendo eco a la iniciativa del Gobierno, y en busca de alternativas que permitieran reducir costos de transporte, decidió experimentar el internamiento de su carga por río el Magdalena, sin embargo De los 180 millones de toneladas de carga que se mueven al año en Colombia, solo un millón 200 mil lo hacen por el Rio Magdalena (Valencia Gil Juan Carlos Renace el transporte de Carga)

La situación de navegabilidad, en el caso de embarcaciones mayores, la longitud navegable es de $10.319 \mathrm{Km}$., de los cuales $6.175 \mathrm{Km}$. (60\%) son permanentes y $4.144 \mathrm{Km}$. (40 \%) transitorios. Por otra parte, para el caso de las embarcaciones menores se cuenta con $18.144 \mathrm{Km}$. de longitud con navegabilidad permanente. Cuenta con 32 puertos de interés nacional, de los cuales 11 están a cargo de Cormagdalena, y 52 puertos, caracterizados por prestar servicios que cubren necesidades de transporte local y regional. La infraestructura portuaria de interés nacional y regional, está compuesta en $57 \%$ por muelles en concreto, $35 \%$ en tierra, $5 \%$ metálicos, $2 \%$ en madera y $1 \%$ por sistema de banda transportadora. De estos terminales, sólo $43 \%$ se encuentra en buen estado, $23 \%$ en regular estado y $34 \%$ en mal estado. (DNP plan de acción para el sector fluvial Documento conpes 2814)

Entre los principales problemas que presenta el sector se citan:

- No cuenta actualmente con la infraestructura portuaria, adecuada y suficiente de centros de transferencia que aseguren las ventajas de un posible desarrollo del transporte multimodal.

- Limitación de las condiciones de navegabilidad de sus principales ríos

- Irregularidad en el servicio, el cual depende de la demanda del servicio.

- Poca atención del gobierno para fortalecer y proteger a las empresas que prestan el servicio.

- Falta de estímulos para desarrollar el comercio y demás actividades económicas. 
- Inexistencia de señalización y balizaje fluvial, la cual no permite la navegación nocturna (Luis Alberto Chávez, Rio Magdalena) (Figuras 3 y 4).

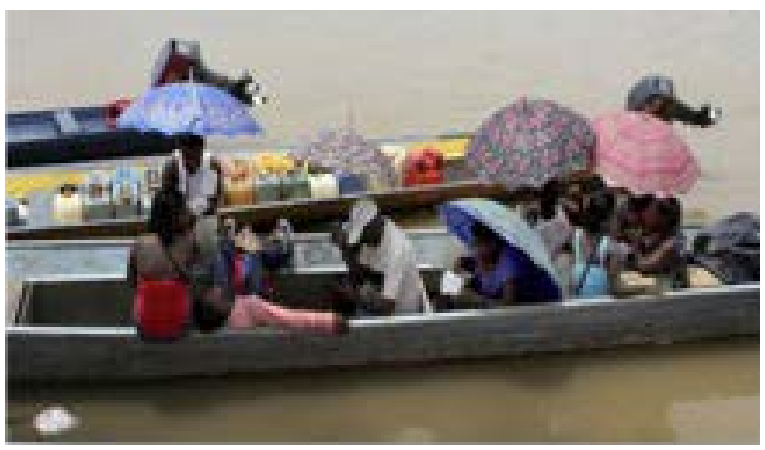

Figura 3. Transporte mixto por el Rio Atrato. Fuente: El Colombiano. Medellín Agosto 11/ 2013

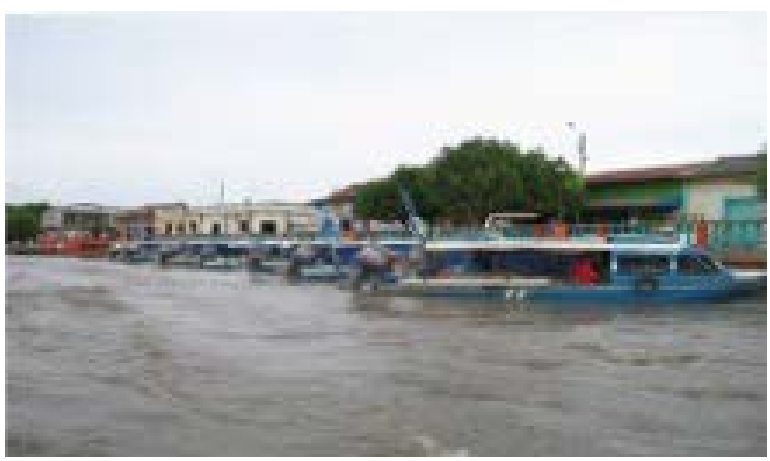

Figura 4. Transporte mixto por el Rio Magdalena. Fuente: NotiCeSar Nov 11/2011

\section{Los contenedores}

Uncontenedor esun recipientedecargaparael transporte aéreo, marítimo, fluvial o terrestre. Las dimensiones del contenedor se encuentran normalizadas para facilitar su manipulación. Por extensión, se llama contenedor a un embalaje de grandes dimensiones utilizado para transportar objetos voluminosos o pesados: motores, maquinaria, pequeños vehículos, etc. Es conocido también con su nombre en inglés, container (Wikipedia Contenedores)

Existen diferentes medidas para contenedores variando en largo y alto:

- El ancho se fija en 8 pies (2,44 metros).

- El alto varía entre 8 pies y 6 pulgadas $(2,59 \mathrm{~m})$ ó 9 pies y 6 pulgadas $(2,89 \mathrm{~m})$.

- El largo varía entre 10 pies (3,04 m); 20 pies (6,08 m); 40 pies $(12,19 \mathrm{~m})$ y 45 pies $(13,71 \mathrm{~m})$.

Lo más extendido a nivel mundial son los equipos de 20 y 40 pies, con un volumen interno aproximado de $32,6 \mathrm{~m} 3$ y $66,7 \mathrm{~m} 3$ respectivamente. Las dimensiones de los contenedores están reguladas por la norma ISO 6346.

La carga máxima puede variar según la naviera y el tipo de contenedor. Los contenedores más normalizados internacionalmente de $20^{\prime}$ tienen un peso bruto máximo de unas $29 \mathrm{t}$ (es decir, la carga más la tara o peso del contenedor) y los de 40' de unas $32 \mathrm{t}$.

Aunque, como muchas veces se traslada el contenedor vía terrestre desde la zona de carga al puerto, hay que atenerse a la legislación vigente en cada país sobre pesos máximos en camiones. La tara o peso del contenedor puede ir de 1,8 thasta $4 \mathrm{t}$ para los de $20^{\prime}$ y de $3,2 \mathrm{t}$ hasta $4,8 \mathrm{t}$ para los de 40 '. (Wikipedia Contenedores)

Tipos de contenedores (fuente: Wikipedia)

Dry Van: Son los contenedores estándar. Cerrados herméticamente y sin refrigeración o ventilación, de 20 y 40 pies.

- Reefer: Contenedores refrigerados de las mismas medidas que el anteriormente mencionado, pero que cuentan con un sistema de conservación de frío o calor y termostato. Deben ir conectados en el buque y en la terminal, incluso en el camión si fuese posible o en un generador externo.

- Open Top: De las mismas medidas que los anteriores, pero abiertos por la parte de arriba. Puede sobresalir la mercancía pero, en ese caso, se pagan suplementos en función de cuánta carga haya dejado de cargarse por este exceso.

- Flat Rack: Carecen también de paredes laterales e incluso, según casos, de paredes delanteras y posteriores. Se emplean para cargas atípicas y pagan suplementos de la misma manera que los open top.

- Open Side: Su mayor característica es que es abierto en uno de sus lados, sus medidas son de 20' o 40'. 
Se utiliza para cargas de mayores dimensiones en longitud que no se pueden cargar por la puerta del contenedor.

- Tank o Contenedor Cisterna: Para transportes de líquidos a granel. Por sus características, solamente se construyen en un tamaño: 20 pies (6 metros) de largo, y 8 pies (2,4 metros) de ancho.

- Flexi-Tank: Para transportes de líquidos a granel. Suponen una alternativa al contenedor cisterna. Un flexi-tank consiste en un contenedor estándar (Dry Van), normalmente de 20 pies, en cuyo interior se fija un depósito flexible de polietileno de un solo uso denominado flexibag.

El papel de los puertos en la cadena de transporte, es hacer más eficiente el sistema de transporte, fomentar el crecimiento del comercio, aliviar la congestión de los principales corredores terrestres, mejorar los enlaces marítimos con las regiones insulares y periféricas, reforzar el transporte multimodal y la logística del transporte En 1977, la Organización Marítima Internacional (OMI), realizó un convenio internacional sobre la seguridad de los contenedores donde dice que el contenedor debe de tener un carácter permanente y uso continuado y un diseño para intercambio de modal rápido, seguro y sencillo. Específica las recomendaciones de seguridad para saber en qué lugar del buque deben ir ciertos tipos de contenedores. Los principales beneficios de los contenedores son la reducción de tiempo en puerto, el incremento en frecuencias de servicio, el aumento en tonelaje por milla, mejor utilización de recursos, seguridad y protección a la carga, estandarización de equipo portuario, desarrollo de industria secundaria y sistematización de servicios (Orillac Rogelio I foro global).

Son muchos los ríos navegables en el mundo, y algunos de los más antiguos están en Europa. En los países ricos los cursos suelen estar acondicionados para facilitar la navegación: rectificación del curso, dragado del fondo, etc. Y también hay canales que conectan unos ríos con otros, para multiplicar la posibilidad de viaje. Para ello es necesario mirar los entornos en que se mueven esos países como el tecnológico, organizacional, ocupacional y especialmente el educativo, que conlleva a los anteriores (Figura 5).

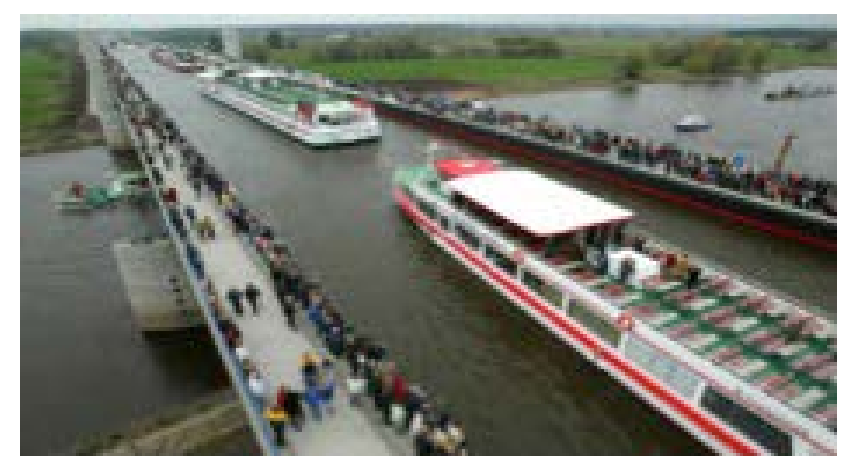

Figura 5. Vías fluviales, embarcaciones, infraestructuras, transporte, sobre el río Elba acorde con las tecnologías de innovación. Fuente: Wasserstrassenkreuz-Magdeburg Oktober/2003

Gran parte del progreso logrado por estos países que conforman el bloque de los más desarrollados en el mundo, ha tenido que ver con su posición geográfica, premiados con costas marinas y ríos, que en hora buena se elevaron a condiciones óptimas para su articulación y aprovechamiento. (La Guía Transporte Fluvial) Colombia a pesar de poseer esas potencialidades, se ha empecinado en ser un país con mentalidad mediterránea, aferrada a un desconocimiento o absurda negativa a disponerse a elevar a condiciones optimas esas evidentes ventajas comparativas, a pesar de crear herramientas como el Documento CONPES 2814 MINTRANSPORTE-DNP: UINFE-DITRAN de 25 de octubre 1995, este CONPES aprobó el "Plan para la Recuperación y Manejo del Río Grande la Magdalena 1995-1998" en el cual se formuló la política que se desarrollará en relación con dicho río, el corredor fluvial más importante del país, a través de la Corporación del Río Grande de la Magdalena, Cormagdalena. Este documento presenta además la estrategia para mejorar la infraestructura fluvial de los canales navegables y terminales portuarios, así como los servicios asociados, en las cuencas fluviales del Magdalena, la Orinoquia, la Amazonia y el Atrato. Incluido en los planes nacionales de desarrollo de los distintos gobiernos hasta la fecha, sin resultados favorables (DNP plan de acción para el sector fluvial Documento conpes 2814).

El Programa de las Naciones Unidas para el Desarrollo (PNUD) establece que la educación de un país es la base para construir una sociedad más justa y sostenible. Tiene un papel central en la reducción de la pobreza, el mejoramiento de las condiciones de vida de las personas y el desarrollo humano. 
Según esto, si una sociedad se caracteriza por la pobreza y presenta grandes inequidades, discriminación y exclusión, esta en el camino equivocado del desarrollo sostenible. Y si un sistema educativo reproduce ese mismo esquema, contribuye a aumentar las diferencias sociales y económicas, por tanto se desvía del camino del progreso equitativo y razonable. Desarrollo sostenible implica la expansión de las libertades y capacidades fundamentales de las actuales generaciones, mientras realizamos esfuerzos razonables para evitar el riesgo de comprometer gravemente, las de las futuras, dice el PNUD. Las sociedades sostenibles están basadas en la creencia de que cada persona tiene derecho a aprender, está en capacidad de contribuir y tiene el compromiso de asegurar que todos los demás compartan el beneficio de la prosperidad social. (PNUD Agenda Siglo XXI).

\section{Conclusión}

Vemos como el entorno de los principales puertos del Caribe Colombiano se mueven en función de las empresas nacionales con vocación exportadora, preparándose para enfrentar los retos que le impondrán los tratados de libre comercio, suscritos en los últimos años, y los que seguramente vendrán en los próximos; acuerdos que podrán cambiar los cimientos de nuestra economía y los modelos de negociación en nuestro país. El plan decenal de educación 2006-2016, contempla la articulación de los medios de educación con los de producción, los mares, ríos, puertos, logística de transporte, distribución física internacional, nuevas tecnologías aplicadas, agroindustria, etc, realizan actividades que requieren estos medios de producción enfocados a la exigencia global de la economía; se hace necesario para las regiones que depende de estos medios de producción, diseñar nuevos programas académicos del perfil que se requiere, de lo contrario es posible que los resultados esperados no sean los más favorables.

\section{Referencias}

Zuidwijk Antonio Puertos y Transporte Intermodal. (Agosto/2010). En:

http://www.antonioz.com.ar/index.php

Orillac Rogelio. (2006). I foro global de logística y Comercio Internacional Contenedores

http://webpicking.com/notas/orillac.htm/

Ospina Hernández, Mariano. (2008). La Navegación Fluvial en Colombia 2008. En:

www.oas.org/cip/docs/areas

Valencia Gil Juan Carlos. (2012). Renace el Transporte de Carga por el Rio Magdalena

$h t t p: / / w w w . e l c o l o m b i a n o . c o m / B a n c o C o n o c i m i e n t o / R /$ renace_el_transporte_de_carga_por_las_aguas_del_rio_ magdalena/renace_el_transporte_de_carga_por_las_aguas_ del_rio_magdalena.asp

Departamento Nacional de Planeación plan de acción para el sector fluvial Documento conpes 2814mintransportednp:uinfe-ditran

ht tps:// www.dnp.gov.co/LinkClick. aspx?fileticket $=c 5$ RjmBiuFLE\%3D\&tabid $=354$
Chávez Luis, Alberto. (2013). Río Magdalena. Revista Logística. En:

www.revistadelogistica.com/Rio-Magdalena.asp

Anaya Tejeros Julio Wikipedia Contenedores Definición/ Clasificación 2009

http://es.wikipedia.org/wiki/Contenedores.

Orillac Rogelio I foro global de logística y Comercio Internacional 2006 Puertos

http://webpicking.com/notas/orillac.htm/

La guía 2000. (2008). Transporte Fluvial. En: http://geografia.laguia2000.com/economia/transporte-fluvial

Departamento Nacional de Planeación. plan de acción para el sector fluvial Documento conpes 2814mintransportednp:uinfe-ditran

ht tps://www.dnp.gov.co/LinkClick. asp $x$ ?fileticket $=c 5$ RjmBiuFLE\%3D\&tabid $=354$

El PNUD "Educación, la agenda del siglo XXI. Hacia un desarrollo humano".

http://www.ing.unal.edu.co/admfac/iei/comunicados/docs/ educacion_agenda_siglo_XXI.pdf. 